\title{
Cementation of proximal femoral nails of the very elderly subject in per-trochanteric fractures
}

\author{
Sorya Plang, Romain Dayan, Frédéric Khiami, Clément Preneau, Olivier Barbier, Camille Choufani
}

From the Clinic of orthopedics and traumatology, Begin Military Hospital, 69 avenue de Paris 94160 Saint-Mandé, France

The management of pertrochanteric fractures (PTF) in the very elderly relies on early verticalisation to limit complications of the decubitus and this requires stable osteosynthesis allowing immediate full support without risk of mechanical failure. The aim of the study was to analyse the value of cementing the cervicocephalic blade during osteosynthesis with a proximal femoral nail.

A prospective bicentric comparative study was conducted. Patients over 90 years of age with PTF were included. Centre A used a PFNA (Proximal Femoral Nail Antirotation) nail without blade cementing and Centre B used the same nail with blade cementing. The primary endpoint was the occurrence of disassembly of the osteosynthesis requiring revision surgery. Secondary endpoints were functional outcome (resumption of walking), postoperative pain and duration of surgery.

Sixty-four patients were included in Centre $A$ and 23 patients were included in Centre $B$. Mean age, gender, and tip-apex distance were comparable between the groups. Postoperative pain and duration of surgery did not show significant differences between the groups. Four patients operated on with an uncemented PFNA $(6.25 \%)$ and one patient operated on with a cemented PFNA nail $(4.35 \%)$ showed early dismantling. The rate of patients returning to walking was significantly higher in the cemented group $(p=0.00005)$.

No significant differences in the rate of dismantling were observed between the two groups. However, the group operated on with a cemented PFNA showed better functional recovery with a significantly higher rate of walking recovery. functional abilities before fracture, fracture type

Keywords : pertrochanteric fracture ; osteosynthesis ; surgery ; elderly.

\section{INTRODUCTION}

The incidence of fractures of the upper extremity of the femur is increasing with the ageing of the population. Pertrochanteric fractures (PTFs) accounted for $36 \%$ of all fractures of the upper extremity of the femur in France in 2009 (1). PTFs are serious : according to the literature, mortality ranges from $10 \%$ to $30 \%$ in the year following the

\footnotetext{
Sorya Plang 1 ,

Romain Dayan²,

Frédéric Khiami ${ }^{2}$,

Clément Preneau ${ }^{1}$,

- Olivier Barbier ${ }^{1,3}$,

- Camille Choufani ${ }^{1}$

${ }^{1}$ Clinic of orthopedics and traumatology, Begin Military Hospital, Saint-Mandé, France.

${ }^{2}$ Orthopaedic Surgery Department, Pitié-Salpêtrière Hospital, Paris, France.

${ }^{3}$ French Military Health Service Academy, Ecole du Val-deGrâce, Paris, France.

Correspondence : Choufani Camille, Clinic of orthopedics and traumatology, Begin Military Hospital, 69 avenue de Paris 94160 Saint-Mandé, France. Fax : 0033 (0) 143985909.

Email : choufanicamille@gmail.com

- 2021, Acta Orthopædica Belgica.
}

No benefits or funds were received in support of this study. None of the authors have a conflict of interest. 
fracture (2-6). They are also functionally serious, as only $25 \%$ of patients regain their previous walking ability (4). PTFs are therefore a major public health issue.

PTFs are extracapsular fractures, which do not involve vascularisation of the femoral head and therefore allow for osteosynthesis. In the elderly, the main challenge of surgery is to obtain a stable osteosynthesis allowing an immediate resumption of walking without the risk of disassembly, which would require complex surgery in fragile patients. The aim is to allow the earliest possible resumption of autonomy. Verticalisation and early mobilisation also make it possible to limit the complications of the decubitus.

Centromedullary nailing (CMN) is an osteosynthesis technique that has the advantage over extramedullary systems, such as plate screws, of medialising and thus reducing the stress forces of the body weight being applied to the osteosynthesis material (7).

The main objective of this study was to compare the Proximal Femoral Nail Antirotation (PFNA) CMN with cementing of the cervicocephalic screw (Fig. 1A) to the PFNA nail without cementing (Fig. 1B) in the occurrence of disassembly, requiring revision surgery, in patients with PTF over 90 years of age. Our hypothesis was that cementing the cervicocephalic screw would result in more stable osteosynthesis with less disassembly, less pain (less
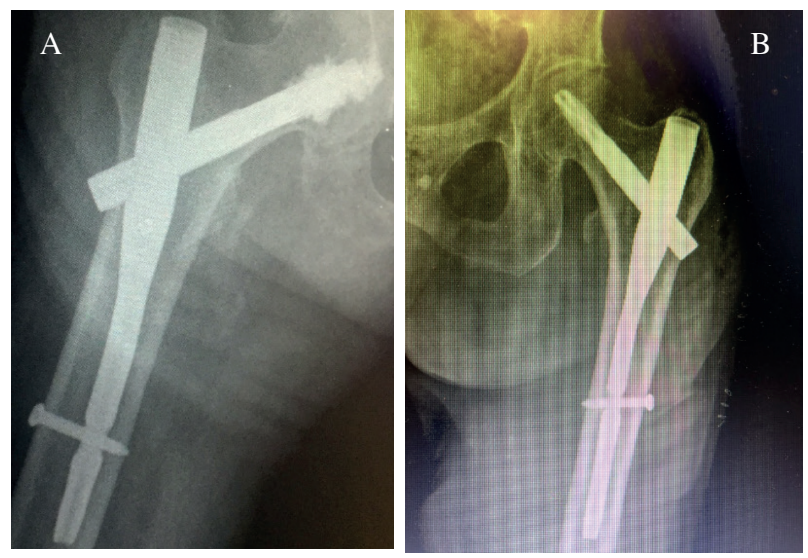

Figures 1. - Proximal medullary nailing of the femur with PFNA : $1 \mathrm{~A}=$ right cemented PFNA $; 1 \mathrm{~B}=$ left uncemented PFNA. need for morphine) and less need for transfusion, without increasing operating time.

\section{MATERIALS AND METHODS}

This is a prospective bicentric multi-operator comparative study. All patients over 90 years of age with radiographically confirmed PTF at Centre A (between 2015 and 2018) and at Centre B (between 2009 and 2016) were included. They had all benefited from osteosynthesis with a PFNAtype nail (Depuy Synthes, Massachusetts, USA). Excluded were patients with pathologic bone fractures, patients treated with screw-plate type osteosynthesis or other CMN systems and those lost to follow-up before the third month postoperatively. In Centre A, the CMN was a non-cemented PFNA. In Centre B, the CMN was a cemented PFNA.

The operators had a junior or senior status. In both groups, this surgery was systematically performed within 24 hours after hospitalization.

The patients all lay supine on an orthopaedic table. Fracture reduction was achieved by external manoeuvring on the orthopaedic table. The quality of the reduction was controlled by an image intensifier used throughout the procedure. The procedure was performed using the standard operating technique.

For blade cementing, a separate cementing kit was provided, separate from the rest of the ancillary instruments and implants. It includes TRAUMACEMTM V+ cement (Depuy Synthes, Massachusetts, USA). After reconstitution, the cement is placed in syringes provided in the kit. Placement of the side-opening cannula into the perforated blade allows cement to be injected around the PFNA blade. TRAUMACEM ${ }^{\mathrm{TM}} \mathrm{V}+$ is a highly viscous PMMA (PolyMethyl MethAcrylate) cement.

After CMN, full support was allowed in all cases. Patients benefited from protocolised clinical and radiological monitoring that was identical in both centres and included instructions for monitoring scarring, pain, quality of walking and radiographs.

\section{a Clinical data}

Patients' age, sex and walking autonomy (before fracture) were recorded.

\section{$b$. The pertrochanteric fracture}


The most recent and widely used classification is that of the AO. It classifies fractures into 3 categories, which are in turn divided into 3 sub-categories. This classification has been used to categorise fractures into stable (A1) and unstable (A2 and A3).

The data collected were :

- Preoperatively : mean age, sex, functional ability before fracture

- Intra-operative : operating time

- Postoperatively:

For each patient an immediate post-operative X-ray was taken. It was used to calculate Baumgaertner's tip-apex distance (TAD) (7), which corresponds to the sum of the distances between the medial end of the screw and the top of the head measured in millimetres on front and side radiographs. The accepted threshold was $25 \mathrm{~mm}$, above which the risk of osteosynthesis dismantling increased.

We recorded for each patient the number of days of morphine consumption and whether or not a blood transfusion was required.

Patients were seen again at 45 days and 3 months post-surgery with pelvic radiographs of the face, hip and side to assess the occurrence of osteosynthesis dislodgement, defined as the scanning of the cervicocephalic screw through the femoral head. Whether or not osteosynthesis dislodgement occurred was the primary endpoint of our study.
The clinical evaluation focused on the possibility of resuming walking with or without technical assistance. A patient was considered to have resumed walking if he or she was able to walk 5 meters with or without technical assistance in the orthopedic consultation room. The classic postoperative monitoring parameters (scar, pain) were checked in order to identify any complications that might have occurred.

The comparative analysis of the two groups was conducted using Fisher's exact test for the qualitative variables. For the comparative analysis of the quantitative variables, a non-parametric test was used (Wilcoxon test). The confidence interval for all tests used was $95 \%$. A value of $p<0.05$ was considered statistically significant.

\section{RESULTS}

The two series of patients were comparable in terms of age, gender and preoperative functional abilities (Table I and II).

No significant difference was found between the two series on the rate of dismantling, the average duration of morphine consumption (days) and the duration of the operation. A statistically significant difference was found for the rate of resumption of walking and the rate of patients transfused postoperatively (Table III).

Table I. - Comparison of preoperative data between the two groups

\begin{tabular}{|l|c|c|c|c|}
\hline & Group A (n=64) & Group B (n=23) & p-value & OR 95\% \\
\hline Walk before & 62 & 22 & 1 & $1,40(0,02 ; 28,21)$ \\
\hline Average age & 93.83 & 92.91 & 0.22 & $1,23(-0,56 ; 2,39)$ \\
\hline Average Tip Apex Distance (mm) & 24.53 & 26.57 & 0.31 & $-1,01(-6,04 ; 1,97)$ \\
\hline Man & 2 & 0 & 1 & Inf $(0,07 ; \inf )$ \\
\hline Woman & 62 & 23 & 1 & Inf $(0,07 ; \inf )$ \\
\hline Stable fracture & 33 & 12 & 1 & $0,98(0,33 ; 2,82)$ \\
\hline Unstable fracture & 31 & 11 & 1 & $1,02(0,35 ; 2,98)$ \\
\hline
\end{tabular}

Table II. - The distribution of the different types of intertrochanteric fractures according to AO in both groups

\begin{tabular}{|c|c|c|c|c|c|c|c|c|c|}
\hline & $\mathrm{A} 1.1$ & $\mathrm{~A} 1.2$ & $\mathrm{~A} 1.3$ & $\mathrm{~A} 2.1$ & $\mathrm{~A} 2.2$ & $\mathrm{~A} 2.3$ & $\mathrm{~A} 3.1$ & $\mathrm{~A} 3.2$ & $\mathrm{~A} 3.3$ \\
\hline $\mathrm{A}$ & 22 & 11 & 0 & 9 & 3 & 6 & 3 & 3 & 7 \\
\hline B & 6 & 4 & 2 & 1 & 3 & 3 & 1 & 0 & 3 \\
\hline
\end{tabular}


Table III. - Comparison of pre- and postoperative data between the two groups

\begin{tabular}{|l|c|c|c|c|}
\hline & Group A (n=64) & Group B (n=23) & P-value & OR 95\% \\
\hline Number and rate of gait recovery postoperatively & $18(28 \%)$ & $18(78 \%)$ & $4.58 \times 10^{-5}$ & $0,112(0,03 ; 0,37)$ \\
\hline Average duration of morphine consumption (days) & 2.39 & 8.09 & 2.64 & $\begin{array}{c}-6,13 \\
(-7,54 ;-3,84)\end{array}$ \\
\hline Number and rate of patients transfused post-operatively & $22(34 \%)$ & $14(61 \%)$ & 0.047 & $0,34(0,11 ; 1,00)$ \\
\hline Average operating time (min) & 43.39 & 47.81 & 0.37 & $\begin{array}{c}-0,83 \\
(-13,90 ; 5,72)\end{array}$ \\
\hline Number of dismantlings & $4(6.25 \%)$ & $1(4.35 \%)$ & 1 & $1,46(0,13 ; 75,44)$ \\
\hline
\end{tabular}

Regardless of the osteosynthesis method used, 4 dismantlings occurred in unstable fractures (A2 or A3 fracture according to the AO classification); thus, dismantling occurred in $11 \%$ of cases with unstable fractures compared to $2 \%$ of cases with stable fractures $(\mathrm{p}=0.15)$.

In Group A, the mean TAD of the patients who did not have their osteosynthesis removed (60 patients) was $29.5 \mathrm{~mm}$, and that of the 4 patients who had their osteosynthesis removed was $24.2 \mathrm{~mm}$ $(\mathrm{p}=0.19)$. In Group B, the mean TAD was $29 \mathrm{~mm}$ for patients who had not had their osteosynthesis removed. The TAD of the patient who underwent disassembly was $26.45 \mathrm{~mm}(\mathrm{p}=0.79)$.

\section{DISCUSSION}

The main objective of this study was to compare the PFNA CMN with cementing of the cervicocephalic screw to the PFNA nail without cementing in the occurrence of disassembly requiring revision surgery in patients with PTF over 90 years of age. We did not find a statistically significant difference in the primary outcome between the two groups, although there was a trend towards a lower rate of disassembly during cementing in the group B. The rate of postoperative recovery of walking was significantly higher in the group with cementing. In an elderly population, whose functional prognosis is based on an early resumption of support, this is an important finding that would need to be confirmed by a later study (8).

Our study shows that cementing the PFNA cervicocephalic screw may be an intriguing option to promote early regaining of support, even though it does not appear to significantly influence the incidence of mechanical failures during PTF CMN osteosynthesis in subjects over 90 years of age.

The TAD was used as a criterion to assess the risk of disassembly of osteosynthesis after PTF CMN. It is the only recognised radiological criterion for assessing this risk (7). The accepted threshold was $25 \mathrm{~mm}$, above which the risk of osteosynthesis disassembly would increase.

The main limitation of this study is the low number of patients included; however, it is to date the only study evaluating for the same osteosynthesis technique a method with cementing and one without cementing of the cervicocephalic screw. The CMN was carried out at two different centres and by different operators. There is therefore a variability between operators and between centres that is difficult to evaluate. Nevertheless, this element makes it possible to consider the results as representative of the current situation. Indeed, in any centre, this type of intervention is carried out in a random way by any of the operators of the same service (often, the young senior operators).

Recent literature highlights the interest in armed osteosynthesis (cementing or other) in terms of reducing the risk of dismantling (9-13), particularly on the osteoporotic bones of elderly subjects. There are many models of $\mathrm{CMN}$, and alternatives to $\mathrm{CMN}$ exist (14-16). However, few studies have analysed the improvement of early support recovery due to the cementing of CMN. In our study, we have chosen to allow full support recovery for all patients because it is a major prognostic factor for their subsequent gain in autonomy. The main therapeutic objective was to resume walking as early as possible. Twenty- 
eight percent of patients resumed walking in group A compared to $78 \%$ in group B at 3 months. This difference is significant. These data are particularly important when one considers the importance of resuming walking in the elderly. However, it is difficult to draw a conclusion based on these results, as this is a secondary endpoint. The functional assessment in our study was limited to resumption of walking with or without technical assistance after surgery. These data are particularly subjective in the population concerned and would need to be confirmed by a study based on objective scores.

In the literature (7), there is a correlation between surgical time and the occurrence of complications such as blood loss, postoperative pain (morphine consumption) and risk of infection ; thus, we chose to evaluate these elements in our follow-up. We found an increase in operating time in group B, but this difference was not significant. No septic complications were observed in group $\mathrm{B}$, and one was observed in group A. Despite the increase in operating time, there was no increase in the rate of postoperative transfusion in the cemented group. On the other hand, morphine consumption was higher in the cemented group (not significant). This was unexpected, as biomechanical studies have shown that cementing would result in greater rigidity, therefore causing less pain due to less stress on the implant (9-11). We have not found any significant argument in the literature that would question cementing as a cause of added pain due to possible cellular necrosis around the cement $(17,18)$.

As quoted in the symposium of the French Society of Orthopaedic Surgery in 2018 on the failures of osteosynthesis, the essential elements to be respected remain the quality of the reduction and the installation of the equipment (with respect to the TAD) (19).

\section{REFERENCES}

1. Oberlin, Mouquet. Les modalités de prise en charge des fractures du col du fémur en France de 1998 à 2009. https:// www.epsilon.insee.fr/jspui/bitstream/1/12499/1/er774.pdf

2. Leboime A, Confavreux CB, Mehsen N, Paccou J, David C, Roux C. Ostéoporose et mortalité. Rev Rhum. 2010 ; 77 : S47-52.
3. Magaziner J, Lydick E, Hawkes W, et al. Excess mortality attributable to hip fracture in white women aged 70 years and older. Am J Public Health. 1997 ; 87 : 1630-1636.

4. Simmermacher RKJ, Ljungqvist J, Bail H, et al. The new proximal femoral nail antirotation (PFNA) in daily practice: results of a multicentre clinical study. Injury. 2008 ; 39 : 932-939.

5. Melton LJ, Achenbach SJ, Atkinson EJ, Therneau TM, Amin S. Long-term mortality following fractures at different skeletal sites: a population-based cohort study. Osteoporos Int J Establ Result Coop Eur Found Osteoporos Natl Osteoporos Found USA. 2013 ; 24 : 1689-1696.

6. Bliuc D, Nguyen TV, Eisman JA, Center JR. The impact of nonhip nonvertebral fractures in elderly women and men. J Clin Endocrinol Metab. 2014 ; 99 : 415-423.

7. Baumgaertner MR, Curtin SL, Lindskog DM. Intramedullary versus extramedullary fixation for the treatment of intertrochanteric hip fractures. Clin Orthop. $1998 ;(348): 87-94$.

8. Kammerlander C, Hem ES, Klopfer T, Gebhard F, Sermon A, Dietrich M, Bach O, Weil Y, Babst R, Blauth M. Cement augmentation of the Proximal Femoral Nail Antirotation (PFNA) - A multicentre randomized controlled trial. Epub 2018. DOI :10.1016/j.injury.2018.04.022

9. Stoffel KK, Leys T, Damen N, Nicholls RL, Kuster MS. A new technique for cement augmentation of the sliding hip screw in proximal femur fractures. Clin Biomech Bristol Avon. 2008 ; 23(1) : 45-51.

10. Von der Linden $P$, Gisep A, Boner V, Windolf $M$, Appelt A, Suhm N. Biomechanical evaluation of a new augmentation method for enhanced screw fixation in osteoporotic proximal femoral fractures. J Orthop Res Off Publ Orthop Res Soc. 2006 ; 24 : 2230-2237.

11. Chirurgie des fractures de l'extrémité proximale du fémur chez les patients âgés. Méthode Recommandations pour la pratique clinique. Décembre 2016. Haute Autorité de Santé. France.

12. Sermon A, Boner V, Schwieger K, et al. Biomechanical evaluation of bone-cement augmented Proximal Femoral Nail Antirotation blades in a polyurethane foam model with low density. Clin Biomech Bristol Avon. 2012 ; 27 : 71-76.

13. Dayan R, Morvan A, Cohen-Bittan J, et al. Étude comparative entre clou PFNA cimenté et clou gamma III dans les fractures per-trochantériennes du sujet âgé. Rev Chir Orthop. 2017 ; 103(7S) : p.S110.

14. Adam $P$. Treatment of recent trochanteric fracture in adults. Oorthop Traumatol Surg Res. 2014 ; 100 : S75-S83.

15. Loubignac F, Chabas JF. A newly designed locked intramedullary nail for trochanteric hip fractures fixation : results of the first 100 Trochanteric implantations. Orthop Traumatol Surg Res. 2009 ; 95 : 139-144.

16. Hélin M, Pelissier A, Boyer P, Delory T, Estellat C, Massin P. Does the PFNATM nail limit impaction in unstable intertrochanteric femoral fracture? A 115 casecontrol series. Orthop Traumatol Surg Res. 2015 ; 101 : 4549. 
17. Boner V, Kuhn P, Mendel T, Gisep A. Temperature evaluation during PMMA screw augmentation in osteoporotic bone-an in vitro study about the risk of thermal necrosis in human femoral heads. J Biomed Mater Res $B$ Appl Biomater. 2009 ; 90 : 842-8.
18. Gundapaneni D, Goswami T. Thermal isotherms in PMMA and cell necrosis during total hip arthroplasty. $J$ Appl Biomater Funct Mater. 2014 ; 12 : 193-202.

19. Bel JC, Féron JM, Loubignac F, et al. Implications techniques et médico-légales des faillites d'ostéosynthèses. Symposium SOFCOT 2018. 